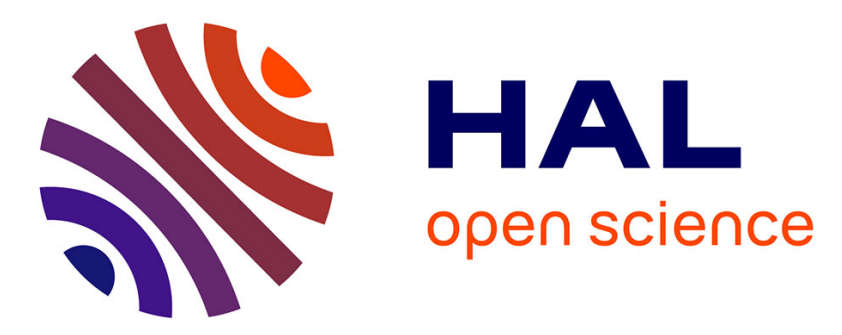

\title{
Étude par bombardement électronique de la durée de vie et de la structure hyperfine des niveau $\mathrm{D}$ du sodium et du césium
}

Y. Archambault, J.P. Descoubes, M. Priou, A. Omont, J.C. Pébay-Peyroula

\section{- To cite this version:}

Y. Archambault, J.P. Descoubes, M. Priou, A. Omont, J.C. Pébay-Peyroula. Étude par bombardement électronique de la durée de vie et de la structure hyperfine des niveau D du sodium et du césium. J. Phys. Radium, 1960, 21 (8-9), pp.677-679. 10.1051/jphysrad:01960002108-9067701 . jpa-00236353

\section{HAL Id: jpa-00236353 https://hal.science/jpa-00236353}

Submitted on 1 Jan 1960

HAL is a multi-disciplinary open access archive for the deposit and dissemination of scientific research documents, whether they are published or not. The documents may come from teaching and research institutions in France or abroad, or from public or private research centers.
L'archive ouverte pluridisciplinaire HAL, est destinée au dépôt et à la diffusion de documents scientifiques de niveau recherche, publiés ou non, émanant des établissements d'enseignement et de recherche français ou étrangers, des laboratoires publics ou privés. 
ETUDE PAR BOMBARDEMENT ÉLECTRONIQUE DE LA DUREE DE VIE

ET DE LA STRUCTURE HYPERFINE DES NIVEAUX D DU SODIUM ET DU CÉSIUM

Par Y. Archambault, J. P. Descoubes,

M. Priou, A. Omont, J. C. Pébay-Peyroula, Laboratoire de Physique de l'École Normale Supérieure.

Par bombardement avec des électrons lents, il est possible de créer des inégalités de population dans les sous-niveaux Zeeman des niveaux atomiques excités et par suite, par résonance magnétique, de déterminẹr dans certains cas favorables leur durée de vie et de mesurer leur structure hyperfine. Cette méthode a été appliquée à différents éléments et décrite dans des publications précédentes $[1,2,3,4]$.

Dans le cas du sodium et du césium, nous avons décelé et identifié diverses résonances. Les différents ensembles de raies ont été isolés à l'aide de filtres colorés Wratten ou à l'aide de filtres interférentiels (tableau I).

TABLEAU I .

\begin{tabular}{|c|c|c|c|c|c|c|}
\hline & & Tra & ITIONS ÉTUDIÉES & & & \\
\hline & $n$ & $n^{2} D_{5 / 2}-m^{2} P_{3 / 2}$ & $n^{2} D_{3 / 2}-m^{2} P_{3 / 2}$ & $n^{2} D_{3 / 2}-m{ }^{2} P_{1 / 2}$ & Niveau étudié & $\begin{array}{l}\text { RAPPORT SIGNAL } \\
\text { SUR BRUIT }\end{array}$ \\
\hline & - & $5 \overline{688^{*} \AA}$ & - & - & $\overline{4^{2}} D_{5 / 2}$ & $\overline{8}$ \\
\hline Sodium & 4 & & 5688 & 5683 & $\left.4^{2} D_{3 / 2}\right)$ & 3 \\
\hline & & $4983 \AA$ & & & $5^{2} D_{5 / 2}$ & 15 \\
\hline & 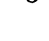 & & 4983 & 4979 & $\left.5^{2} D_{3 / 2}\right)$ & 6 \\
\hline & & $6983 \AA$ & & & $7^{2} D_{5,2}$ & 10 \\
\hline • & $\gamma$ & & 6973 & 6723 & $\left.7^{2} D_{3 / 2}\right\}$ & 5 \\
\hline & 0 & $6217 \AA$ & & & $8 .{ }^{2} D_{5 / 2}$ & 10 \\
\hline & 0 & & 6213 & 6010 & $\left.8^{2} D_{3 / 2}\right)_{\text {, }}$ & 5 \\
\hline Césium & 9 & $5847 \AA$ & & . & $9^{2} D_{5 / 2}$ & 10 \\
\hline réf. [6] & & & 5844 & 5663 & $\left.9^{2} D_{3 / 2}\right)$ & 3 \\
\hline & 10 & $5635 \AA$ & & & $10^{2} D_{5 / 2}$ & 8 \\
\hline & 10 & & 5636 & 5466 & $\left.10^{2} D_{3 / 2}\right\}$ & 3 \\
\hline & 14 & $5503 \AA$ & & & $\left.11^{2} D_{5 / 2}\right\}$ & 8 \\
\hline & 11 & & 5503 & 5341 & $\left.11^{2} D_{3 / 2}\right)$ & non observé \\
\hline
\end{tabular}


La figure 1 donnezen fonction du champ magnétique, les niveaux d'énergie Zeeman issus d'un niveau ${ }^{2} D_{5 / 3}$ du sodium. Les flèches montrent les différentes transitions que l'on est susceptible d'observer par résonance magnétique.

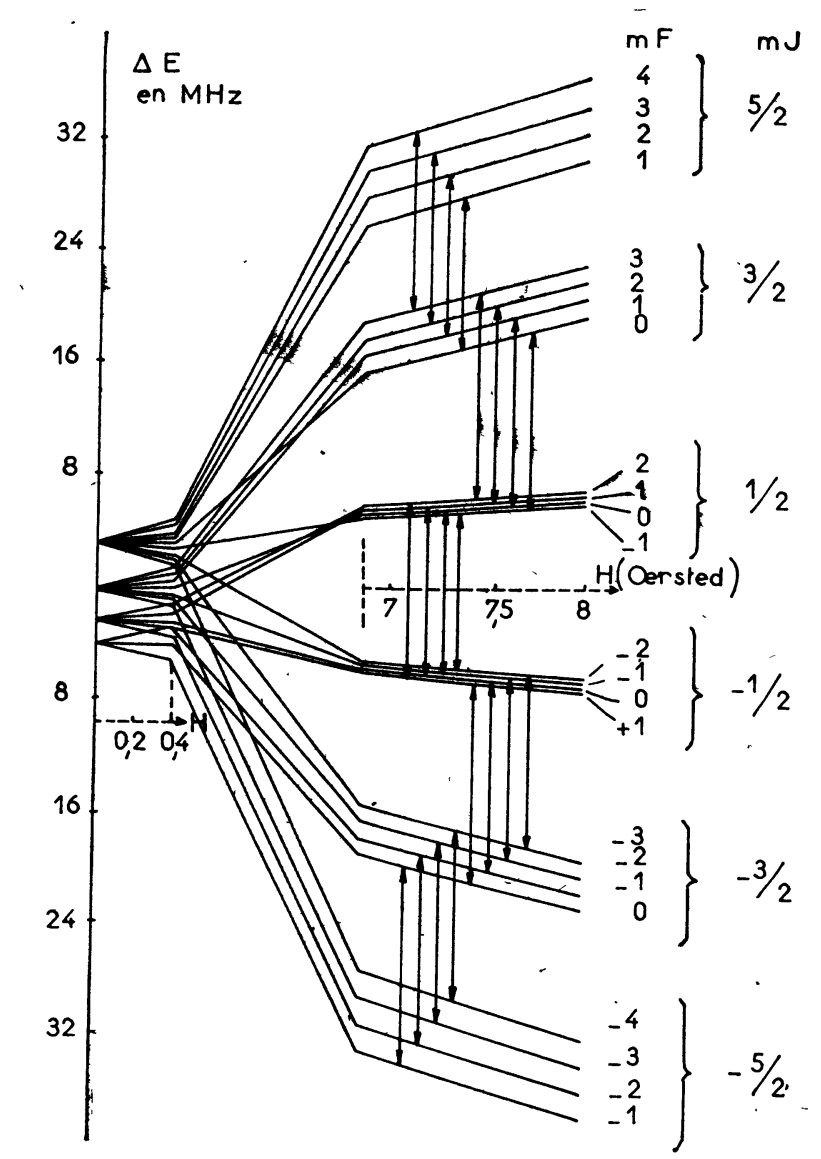

FIG. 1. - Décomposition Zeeman du niveau ${ }^{2} D_{5 / 2}(I=3 / 2)$ du sodium. (Pour permettre un dessin lisible, nous avons pris $a=0,8 \mathrm{Mc} / \mathrm{s})$.

Pour ces différentes résonances, les premières expériences ont été faites en balayage de champ magnétique à une fréquence fixe de 25 ou $50 \mathrm{Mc} / \mathrm{s}$. On a observé une raie unique résultante des différentes transitions. La position de cette raie montre, d'après la valeur du facteur de Landé, que l'on est dans la région de découplage $I J$ total. La structure hyperfine est donc beaucoup plus petite que $25 \mathrm{Mc} / \mathrm{s}$ et est, d'autre part, du même ordre de grandeur que la largeur naturelle du niveau ou plus petite.

Notre but fut de tirer le maximum de renseignements de la forme de ces raies de résonance. Il est apparu que le balayage en champ magnétique à fréquence fixe apportait, par suite du découplage $I J$, des distorsions importantes aux courbes de résonance. Nous avons donc réalisé un dispositif permettant d'observer les résonances en balayage de fréquence.

Il est nécessaire d'avoir un champ oscillant $H_{1}$ dont l'amplitude soit de l'ordre du Gauss et soit indépen- dante de la fréquence. Une puissance de l'émetteur, de l'ordre du watt, est nécessaire. L'amplitude du ba- layage doit en outre être assez grande pour décrire entièrement une résonance. L'émetteur utilisé est représenté par la figure 2 . Les bobines créant le champ

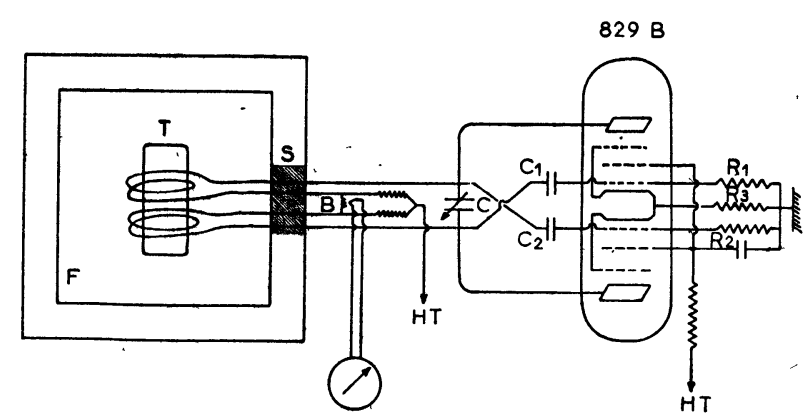

Fig. 2. - Schéma de principe du.montage.

$F=$ four

$\mathrm{S}=$ passage isolant en stéatite,

$\mathrm{T}=$ cellule àłbombardement électronique.

$\mathrm{B}=$ bolomètre

$\mathrm{C}_{1}, \mathrm{G}_{2}=15 \mathrm{pF}$.

$R_{1}, R_{2}=1 \mathrm{k} \Omega$.

$\mathbf{G}=$ condensateur variable $20-700 \mathrm{pF}$ (permet un balayage de 8 à $20 \mathrm{Mc} / \mathrm{s}$ ).

de radiofréquence servent de circuit accordé à l'émetteur. La fréquence de l'émetteur est modifiée en agissant seulement sur le condensateur $\mathrm{C}$ qui est entrainé par un moteur synchrone. Un bolomètre à thermocouple permet de maintenir constant (en variant la haute tension appliquée à l'émetteur) le courant dans les bobines et par suite le champ magnétique de haute fréquence. Des esşais de servomécanisme pour maintenir ce niveau constant se sont révélés très décevants, à cause de la constante de temps introduite par l'inertie thermique du bolomètre. Un réglage manuel a été finalement adopté.

La mise en œuvre de cette technique de balayage de fréquence s'ẹst révélée difficile, car le balayage de fréquence produit au niveau des électrodes de la cellule un champ électrique variable et l'intensité lumineuse émise varie avec la fréquence. De nombreuses distorsions et résonances parasites apparaissent. Nous avons pu les éliminer par une étude soignée de la géométrie des électrodes et du système optique [5]. Ces différentes résonances ont été observées avec un rapport signal sur bruit de l'ordre de 10 (cf. tableau I).

Connaissant les équations donnant en fonction du champ et de la fréquence les différents niveaux d'énergie de la figure 1, il est algébriquement possible de déterminer un ordre de grandeur de la largeur limite naturelle $\Delta \omega_{1}$ du niveau étudié et de la constante de structure hyperfine $a$, en étudiant de façon systématique la position et la largeur des résonances observées à des fréquences différentes. Ceci suppose en outre la connaissance de la répartition de population créée par le bombardement éleotronique dans les différențs sousniveaux.

En pratique, la détermination que l'on peut obtenir ainsi est très peu précise, les variations de facteur de Landé et de largeur étant très faibles dans le domaine où l'observation des résonances est possible. Ces résonances ont une largeur de l'ordre de quelques mégacycles et on ne peut les observer que lorsque la fré- 
quence est supérieure à leur largeur. En outre, dans le cas du sodium, les structures fines $D_{5 / 2}-D_{3 / 2}$ sont très faibles (\# $700 \mathrm{Mc} / \mathrm{s}$ pour le niveau $5^{2} D_{5 / 2}$ ) et le découplage $L S$ devient appréciable lorsque la fréquence de résonance dépasse $50 \mathrm{Mc} / \mathrm{s}$.

Par ailleurs, nous n'avons qu'une connaissance qualitative des populations relatives des sous-niveaux Zeeman [2], ce qui est la cause essentielle de l'imprécision des résultats que nous avons obtenus actuellement :

Sodium-Niveau $5{ }^{2} D_{5 / 2}$

$$
\begin{gathered}
a<0,33 \mathrm{Mc} / \mathrm{s} \\
2 \cdot 10^{-7} \mathrm{~s}<\tau<4,6 \cdot 10^{-7} \mathrm{~s} .
\end{gathered}
$$

Césium-Niveau $9{ }^{2} D_{5 / 2}$

$$
0,195 \mathrm{Mc} / \mathrm{s}<a<0,45 \mathrm{Mc} / \mathrm{s}
$$$$
\tau=(0,95 \pm 0,1) 10^{-7} \mathrm{~s} \text {. }
$$

Lettre reçue le 8 juillet 1960.

\section{BIBLIOGRAPHIE}

[1] Pébay-Peyroula (J. G.), J. Physique Rad., 1959, 20, 669.

[2] Pébay-Peyroula (J. C.), J. Physiquie Rad., 1959, 20, 721.

[3] Descoubes (J. P.) et Pébay-Peyroula (J. G.), $C . R$. Acad. Sc., 1958, 247, 2330.

[4] May (A. D.), C. R. Acad. Sc., 1960, 250, 3616

[5] Archambault (Y.), Thèse' 3 e cycle, Paris, 1960.

[6] Priou (M.), Diplôme d'Études Supérieures, Paris, 1960. 